\title{
Power Allocation for Capacity Maximization in Eigen-Mimo With OUTPUt SNR Constraint
}

\author{
S. Alireza Banani ${ }^{1}$ and Dena Homayounieh ${ }^{2}$ \\ ${ }^{1}$ Department of Electrical and Computer Engineering, University of Toronto, Toronto, \\ ON, Canada \\ ${ }^{2}$ Faculty of Science and Engineering, York University, Toronto, ON, Canada
}

\begin{abstract}
Water-filling is the power allocation that maximizes the parallel-channel eigen-MIMO informationtheoretic capacity. However, for practical designs, designing for error performance is also important. The overall uncoded error rate, in a system with water-filled eigen-channels using digital modulation, degrades through the errors in the data that uses the weaker eigen-channels. This is because in water-filling, a lower transmit power is allocated (and a lower proportion of the overall capacity) to the weaker eigenchannels. One general solution is to discard the weaker eigen-channels, but this does still not allow simple control over the values of, or trade-off between, capacity and the error rate. In this paper a design approach is presented for the control of, and improvement in, the overall error performance, while maintaining the ergodic capacity as high as possible. The approach is direct; the eigen-channel power allocation is optimized for capacity maximization constrained by a desired error performance on the eigenchannels via constraints on their SNRs. A useful feature is that the capacity and the error performance can be managed through the eigen-channel SNR constraint. Statistical simulations with Rayleigh channels quantify the significant SER improvement compared to full eigen-MIMO (maximum number of eigen-channels) with water-filling, when using a fixed modulation set. This set comprises pre-chosen constellations for a constant number of eigen-channels, and this remains fixed for the all the channel realizations, i.e., with time for the time-varying channel. The improvement in error performance is at the expense of a small loss in capacity.
\end{abstract}

\section{KEYWORDS}

MIMO; water-filling; convex optimization; eigen-channel; ergodic capacity; MIMO capacity

\section{INTRODUCTION}

Open-loop multiple-input multiple-output (MIMO) systems have the practical advantage of not requiring channel knowledge at the transmitter. But this practical advantage comes with a performance penalty because joint optimization between the transmitter and receiver is not fully deployed. When channel state information (CSI) is available at the transmitter, as assumed in this paper, MIMO performance can be improved based on a desired optimization criterion such as capacity, average error rate, etc.

Several jointly optimal linear precoder and decoder (LPD) designs, also called beamformers, based on perfect channel knowledge at the transmitter, are presented in the literature. These are too numerous to list here, but representative examples are [1]-[12]. The work in [1] includes the design that maximizes output SNR, which is called dominant eigenmode transmission. It 
transmits only via the strongest eigenchannel, so the power allocation is trivial and the design is very practical. Water-filled power allocation (i.e., with the total power constrained) for capacity maximization, is presented in [2] and [3]. For Rayleigh channels, the majority of the available capacity is available by deploying just one or a few of the available eigenchannels [4]. The optimal design that minimizes the overall error rate allocates the power on the eigenchannels according to an inverse water-filling policy [5], [6]. A different approach based on inverse SNR (or equivalently, mean squared estimation error) between the input and output of each eigenchannel is studied in [5], [7]. A general solution is a weighted sum of inverse SNRs and leads to a number of well-known solutions depending on the choice of weights [7]. The optimum design that maximizes SNR subject to a zero-forcing constraint is also considered in [6]. An optimal LPD design in [8] is for minimizing the pairwise error probability (PEP) with the total transmit power constraint. Minimization of the geometric mean square error, defined as the determinant of the error covariance matrix, is treated in [9]. Capacity maximization with a peak power constraint is treated in [10].

There are many other specialized schemes (e.g. [11]-[14] with channel estimation and imperfect feedback), but the basic principles appear in different forms from different disciplines (information theory, signal processing, communications theory and techniques, adaptive antennas, etc.). However, there are practical shortcomings with each type of optimized LPD design. For offering insight into MIMO configurations that are practical but can still maintain high performance, formulations are required that lie between the limiting capacity, and, for example, the existing constrained design examples of [1]-[12].

As an example, using water-filling in practice (i.e., with digital modulations), causes the uncoded error performance to deteriorate with the weakest eigenchannel. Although the weaker eigenchannels contribute weakly to the overall capacity, the large difference between the average SNR of the strongest eigenchannel and the average SNR on the lowest eigenchannel can cause disproportionate error rates across the eigen-channels. In this sense, the overall error rate is dominated by the weakest eigen-channel. For example, in a $3 \times 3$ system with water-filling, the difference between the average SNR on the strongest eigenchannel and the average SNR on the lowest eigenchannel is approximately $13.5 \mathrm{~dB}$ when the average SNR at each receive antenna is $20 \mathrm{~dB}$. This difference increases to $16.8 \mathrm{~dB}$ in a $4 \times 4$ system. As a result, full eigen-MIMO systems with water-filling (i.e. with maximized capacity without an error performance constraint) have a weak uncoded SER performance which is may not be desirable in practice.

A preferred system has good reliability and high throughput, simultaneously. One solution is to deploy only the strong eigen-channels, but it is still not straightforward to control the capacity and SER. Also, the highest possible capacity cannot always be guaranteed for a desired output SER performance - it is well-known that these quantities trade-off with each other. For the simple example of a $2 \times 2$ MIMO with the fixed modulation set (see below) and a target overall SER of $10^{-3}$, the dominant eigenmode transmission is not the scheme for the highest capacity (seen via simulation below, for $25 \mathrm{~dB}$ SNR).

In this paper, the capacity and reliability (the uncoded SER), are determined together. The method is to constrain the eigenchannel SNRs for reliability (maximum error rate) and seek a power allocation over the eigenchannels for the highest capacity. First, an optimization problem is addressed for maximizing the capacity with the total transmit power constraint and a maximum allowable SNR reduction (loss of SNR relative to known average SNR at each receiver) imposed on the eigenchannels - a quantity controlled by the system designer. The optimal power allocation is obtained via convex optimizations with inequality constraints [16]. The use of convex optimization itself is not new - it has become a standard tool in MIMO systems design over the last decade. Then, based on the SNR at each receiver and the maximum allowable SNR loss, the 
best power allocation scheme is selected from the presented optimization, water-filling and dominant eigenmode transmission for the highest capacity. One feature of the approach is that the position of the capacity between the bounds can be controlled by appropriately setting the maximum allowable SNR loss.

The simulation results demonstrate an improvement in the SER performance over the case with water-filling, using a fixed modulation set in a $2 \times 2$ MIMO system (a choice of QAM is assigned to eigenchannel 1 and BPSK to eigenchannel 2). In general, fixed-set modulation has different constellations assigned to a fixed number of eigenchannels, all the time. Fixed-common modulation, in which the same constellation is assigned to all the eigenchannels, is a special case of fixed-set modulation. In other words, unlike adaptive modulation, the modulation over the ordered eigenchannels does not change for different channel realizations. The advantage of the fixed-set modulation over adaptive modulation is the reduced complexity in both hardware and required protocol support. The setting-up of adaptive modulation, particularly in a large multiuser network, can lead to a large capacity overhead in the system, e.g. [15]. Finally, in discussing reliability via the error performance of a practical system, the role of data coding is important, but it is not yet possible to optimize eigen-MIMO with general coding.

The rest of the paper is organized as follows. Section II describes the eigen-MIMO system model. The problem of capacity maximization with SNR constraint is addressed in section III, with the simulation results presented in section VI, and section V is the conclusion. The notation is conventional: vectors are lower case letters and matrices are in bold upper case, with $(\cdot)^{H},(\cdot)^{T}$ and $(.)^{*}$ meaning conjugate transpose, transpose, and complex conjugate, respectively; $\mathbf{I}$ is the identity matrix; and $E\{\cdot\}$ denotes expectation.

\section{Closed-LoOP MIMO SySTEM MODEL}

Consider a MIMO quasi-static, flat block-fading channel with $M$ transmit and $N$ receive antennas. The channel is modeled by a random (fading) distribution which remains static over a fading block cycle, but becomes independent across different blocks. A beamformer $\mathbf{W}$, derived using channel knowledge at the transmitter, is shown in Fig. 1.

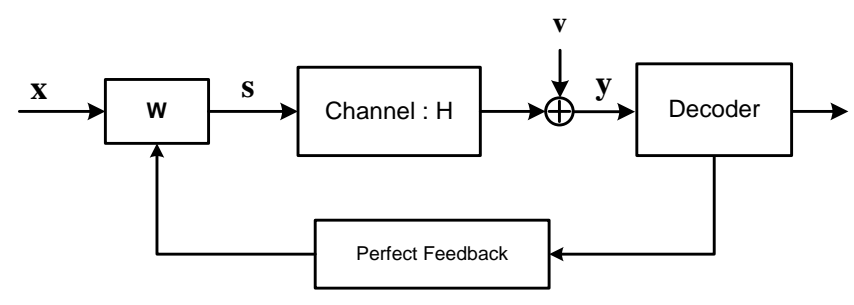

Figure 1. An ideal, closed-loop MIMO system with a transmit beamformer for using channel knowledge which has ideal feedback from the receiver. The ideal feedback link is not part of the capacity calculation for the forward link.

At each symbol time, the $M \times 1$ data vector signal to be transmitted, $\mathbf{x}$, is multiplied by the $M \times M$ weight matrix $\mathbf{W}$, before transmission. With perfect timing, etc., the MIMO system is modeled in the usual way with notation

$$
\mathbf{y}=\sqrt{\frac{\rho}{M}} \mathbf{H W} \mathbf{x}+\mathbf{v}=\sqrt{\frac{\rho}{M}} \mathbf{H s}+\mathbf{v}
$$


where $\mathbf{y}$ is the $N \times 1$ received signal vector, $\mathbf{H}$ is the channel matrix with $(\mathbf{H})_{i, j} \sim C N(0,1), \rho$ is the known average SNR at each receive antenna, and $\mathbf{v} \sim C N(0,1)$ is the additive noise. With $E \mathbf{x x}^{H}=\mathbf{I}_{M}$ and $\mathbf{s}=\mathbf{W} \mathbf{x}$, the covariance matrix of the transmitted signal is given by $\mathbf{R}_{\mathrm{ss}}=E \mathbf{s} \mathbf{s}^{H}=\mathbf{W} \mathbf{W}^{H}$. From the total average transmit power constraint, $\mathbf{W}$ satisfies $\|\mathbf{W}\|_{F}^{2}=\operatorname{tr} \mathbf{R}_{\mathrm{ss}}=M$, so with $\mathbf{s}=\mathbf{W} \mathbf{x}$, we have $E \operatorname{trs\mathbf {s}^{H}}=M$.

\section{EIGEN-MIMO CAPACITY MAXIMIZATION}

The capacity of a sample realization of the channel is first addressed. The ergodic capacity is the ensemble average of the capacity achieved when the optimization is performed for each realization of $\mathbf{H}$, i.e. $C=E C_{\mathbf{H}}$. The information theoretic capacity with a fixed channel $\mathbf{H}$ is defined as [3]

$$
C_{\mathbf{H}}=\max _{\left.p_{\mathbf{s}} \mathbf{s}\right)} I(\mathbf{s} ; \mathbf{y})=\max _{E \operatorname{trs}^{H}=M} \log _{2} \operatorname{det}\left(\mathbf{I}_{N}+\frac{\rho}{M} \mathbf{H R}_{\mathbf{s s}} \mathbf{H}^{H}\right)
$$

where $p_{\mathbf{s}}(\mathbf{s})$ is the probability density function of the vector $\mathbf{s}$, and $I(\mathbf{s} ; \mathbf{y})$ is the mutual information between $\mathbf{s}$ and $\mathbf{y}$.

The matrix $\mathbf{H}$ with rank $r=\min (M, N)$ can be rewritten as $\mathbf{H}=\mathbf{U} \boldsymbol{\Lambda}^{1 / 2} \mathbf{V}^{H}$ where $\mathbf{V}$ and $\mathbf{U}$ are the unitary matrices containing the corresponding input and output singular vectors, respectively and $\boldsymbol{\Lambda}^{1 / 2}$ is a non-negative $N \times M$ diagonal matrix with $i$ th diagonal element as $\lambda_{i}^{1 / 2}$ (the square root of $i$ th eigenvalue). In addition the diagonal elements satisfy $\lambda_{i} \geq \lambda_{i+1}$. As a result, $\mathbf{H}^{H} \mathbf{H}=\mathbf{V} \Lambda \mathbf{V}^{H}$, and

$$
C_{\mathbf{H}}=\max _{E \text { tr s }^{H}=M} \log _{2} \operatorname{det}\left(\mathbf{I}_{N}+\frac{\rho}{M} \boldsymbol{\Lambda}^{1 / 2} \mathbf{V}^{H} \mathbf{R}_{\mathbf{s s}} \mathbf{V} \boldsymbol{\Lambda}^{1 / 2}\right)
$$

Also, note that $\tilde{\mathbf{R}}_{\mathrm{ss}}=\mathbf{V}^{H} \mathbf{R}_{\mathrm{ss}} \mathbf{V}$ is non-negative definite, and $\operatorname{tr} \tilde{\mathbf{R}}_{\mathrm{ss}}=\operatorname{tr} \mathbf{R}_{\mathrm{ss}}$. Thus, the maximization over $\mathbf{R}_{\mathrm{ss}}$ with $E \operatorname{trss} \mathbf{s}^{H}=M$, can be over $\tilde{\mathbf{R}}_{\mathrm{ss}}$ with $E \operatorname{tr} \widetilde{\mathbf{s}} \widetilde{\mathbf{s}}^{H}=M$. Moreover, since for any non-negative definite matrix $\mathbf{A}, \operatorname{det} \mathbf{A} \leq \prod_{i}[\mathbf{A}]_{i, i}$, so

$$
\operatorname{det}\left(\mathbf{I}_{N}+\frac{\rho}{M} \boldsymbol{\Lambda}^{1 / 2} \widetilde{\mathbf{R}}_{\mathrm{ss}} \boldsymbol{\Lambda}^{1 / 2}\right) \leq \prod_{i}\left(1+\frac{\rho}{M}\left[\widetilde{\mathbf{R}}_{\mathrm{ss}}\right]_{i, i} \lambda_{i}\right)
$$

with the equality for when $\widetilde{\mathbf{R}}_{\mathrm{ss}}$ is diagonal. Therefore

$$
C_{\mathbf{H}}=\max _{E \operatorname{tr}_{\tilde{\mathbf{s}} \widetilde{s}^{H}=M}} \sum_{i=1}^{r} \log _{2}\left(1+\frac{\rho}{M} p_{i} \lambda_{i}\right)
$$

where $p_{i}=\left[\tilde{\mathbf{R}}_{\mathrm{ss}}\right]_{i, i}$, and the optimum design corresponds to the SNR on the eigenchannels. The above results set the scene and are known. The remaining problem is the optimal power allocation under SNR constraint. 


\subsection{Optimal Power with SNR Constraint}

Here, the $2 \leq L \leq r$ strongest eigenchannels are deployed, and their SNRs are constrained as

$$
S N R_{j}(\mathrm{~dB}) \geq(\underbrace{\rho(\mathrm{dB})-\delta_{S N R}(\mathrm{~dB})}_{\xi(\mathrm{dB})}) ; j=1, \ldots, L
$$

where $\delta_{S N R}$ is the maximum difference between the SNR on the eigenchannels and the average SNR at each receive antenna, $\rho$. In other words, the approach guarantees that the SNR loss on each eigenchannel is smaller than $\delta_{S N R}$. Thus, in this optimization problem, there are $L$ inequality constraints, $(\rho / M) p_{j} \lambda_{j} \geq \eta=10^{\xi(\mathrm{dB}) / 10}$, in (6) as well as one equality constraint, $\sum p_{i}=M$. The optimization problem now becomes

$$
\begin{array}{cl}
\underset{p_{1}, p_{2}, \ldots, p_{L}}{\operatorname{maximize}} & f_{0}\left(p_{1}, p_{2}, \ldots, p_{L}\right)=\sum_{i=1}^{L} \log _{2}\left(1+(\rho / M) p_{i} \lambda_{i}\right) \\
\text { subject to } & (\rho / M) p_{j} \lambda_{j} \geq \eta ; j=1, \ldots, L \\
& \sum_{i=1}^{L} p_{i}=M
\end{array}
$$

where the objective for the maximization, i.e., $\sum_{i=1}^{L} \log _{2}\left(1+(\rho / M) p_{i} \lambda_{i}\right)$, is concave in the variable $p_{i} ; i=1, \ldots, L$. Equivalently, we can rewrite (7) as

$$
\begin{aligned}
& \underset{p_{1}, p_{2}, \ldots, p_{L}}{\operatorname{minimize}} f_{0}\left(p_{1}, p_{2}, \ldots, p_{L}\right)=-\sum_{i=1}^{L} \log _{2}\left(1+(\rho / M) p_{i} \lambda_{i}\right) \\
& \text { subject to } f_{j}\left(p_{1}, p_{2}, \ldots, p_{L}\right)=\eta-(\rho / M) p_{j} \lambda_{j} \leq 0 ; j=1, \ldots, L \\
& \quad \sum_{i=1}^{L} p_{i}=M
\end{aligned}
$$

where $f_{1}, f_{2}, \ldots, f_{L}$ are convex real functions of $p_{i} ; i=1, \ldots, L$. In fact, (8) is a convex optimization problem that includes inequality constraints. A particular interior-point algorithm called the logarithmic barrier method [16] is used here to solve the problem (8). The first step is to rewrite (8), making the inequality constraints implicit in the objective

$$
\begin{aligned}
& \underset{p_{1}, p_{2}, \ldots, p_{L}}{\operatorname{minimize}} f_{0}\left(p_{1}, p_{2}, \ldots, p_{L}\right)+\sum_{j=1}^{L} g\left(f_{j}\left(p_{1}, p_{2}, \ldots, p_{L}\right)\right) \\
& \text { subject to } \sum_{i=1}^{L} p_{i}=M
\end{aligned}
$$

where $g: \mathfrak{R} \rightarrow \mathfrak{R}$ is the indicator function for the nonpositive reals,

$$
g(u)=\left\{\begin{array}{lll}
0 & ; & u \leq 0 \\
\infty & ; & u>0
\end{array} .\right.
$$

The problem (9) has no inequality constraint, but its objective function is not differentiable, so descent methods (e.g, Newton's method, etc.) cannot be applied. The logarithmic barrier method approximates the indicator function, $g$, by the function

$$
\hat{g}(u)=-(1 / t) \log _{10}(-u)
$$


where $t>0$ is a parameter that sets the accuracy of the approximation. Like $g$, the function $\hat{g}$ is convex and nondecreasing, and becomes $\infty$ for $u>0$. Unlike $g$, however, $\hat{g}$ is differentiable and increases to $\infty$ as $u$ increases to zero. As $t$ increases, the approximation becomes more accurate. Substituting $\hat{g}$ for $g$ in (9) gives

$$
\begin{aligned}
& \underset{p_{1}, p_{2}, \ldots, p_{L}}{\operatorname{minimize}} f_{0}\left(p_{1}, p_{2}, \ldots, p_{L}\right)+(1 / t) \sum_{j=1}^{L}-\log _{10}\left(-f_{j}\left(p_{1}, p_{2}, \ldots, p_{L}\right)\right) \\
& \text { subject to } \sum_{i=1}^{L} p_{i}=M
\end{aligned}
$$

The objective here is convex since $-(1 / t) \log _{10}(-u)$ is convex and increasing in $u$, and it is differentiable. The function $\phi\left(p_{1}, p_{2}, \ldots, p_{L}\right)=-\sum_{j=1}^{L} \log _{10}\left(-f_{j}\left(p_{1}, p_{2}, \ldots, p_{L}\right)\right)$ is called the logarithmic barrier or log barrier for the problem (12). Its domain is the set of points that satisfy the inequality constraints of (8) strictly. Denoting $\left(p_{1}^{\text {opt }}, p_{2}^{\text {opt }}, \ldots, p_{L}^{\text {opt }}\right)$ as the solution of (12), it is shown [16; page 563] that $\left(p_{1}^{\text {opt }}, p_{2}^{\text {opt }}, \ldots, p_{L}^{\text {opt }}\right)$ is no more than $L /$-suboptimal. This suggests a straightforward method for solving the original problem (8), with a specified accuracy $\varepsilon$ by taking $t=L / \varepsilon$.

The next step is to solve the equality constraint problem (12), by eliminating the equality constraint and then solve the resulting unconstrained problem using methods for unconstrained minimization. $p_{1}$ (for example) can be eliminated using the parameterization, $p_{1}=M-\sum_{i=2}^{L} p_{i}$. The reduced problem is then

$$
\begin{aligned}
& \underset{p_{2}, p_{3}, \ldots, p_{L}}{\operatorname{minimize}} \psi\left(p_{2}, p_{3}, \ldots, p_{L}\right) \\
& =-t\left[\log _{2}\left(1+(\rho / M) \lambda_{1}\left(M-\sum_{i=2}^{L} p_{i}\right)\right)+\sum_{i=2}^{L} \log _{2}\left(1+(\rho / M) p_{i} \lambda_{i}\right)\right] \\
& -\log _{10}\left((\rho / M) \lambda_{1}\left(M-\sum_{i=2}^{L} p_{i}\right)-\eta\right)-\sum_{j=2}^{L} \log _{10}\left((\rho / M) p_{j} \lambda_{j}-\eta\right)
\end{aligned}
$$

where the objective is now multiplied by $t$. Since an affine function of a convex function is also convex, eliminating equality constraints preserves convexity. Moreover, the elimination of the equality constraints involves linear algebraic operations. Thus, (13) and (12) are equivalent. Since $\psi\left(p_{2}, p_{3}, \ldots, p_{L}\right)$ is differentiable, a sufficient condition for a point $\left(p_{2}^{\text {opt }}, p_{3}^{\text {opt }}, \ldots, p_{L}^{\text {opt }}\right)$ to be optimal is

$$
\nabla\left(\psi\left(p_{2}^{\mathrm{opt}}, p_{3}^{\mathrm{opt}}, \ldots, p_{L}^{\mathrm{opt}}\right)\right)=0
$$

where $\nabla($.$) denotes the gradient operator. Thus solving the unconstrained minimization$ problem (13) is the same as finding a solution of (14), which is a set of $L-1$ equations in the $L-1$ variables $p_{2}, p_{3}, \ldots, p_{L}$. There is no analytical solution to the optimality problem (14) and so the problem is solved iteratively, for example using gradient descent:

$$
\mathbf{p}^{(n+1)}=\mathbf{p}^{(n)}-\mu^{(n)} \nabla \psi
$$

where $\mathbf{p}^{(n)}=\left[\begin{array}{llll}p_{2}^{(n)} & p_{3}^{(n)} & \cdots & p_{L}^{(n)}\end{array}\right]^{T}$ is the solution vector point at $n$th iteration and 


$$
\begin{aligned}
{[\nabla \psi]_{k-1,1}=\frac{\partial \psi}{\partial p_{k}}=} & -\frac{t}{\log _{10}^{2}}\left(\frac{-(\rho / M) \lambda_{1}}{1+(\rho / M) \lambda_{1}\left(M-\sum_{j=2}^{L} p_{j}\right)}+\frac{(\rho / M) \lambda_{k}}{1+(\rho / M) p_{k} \lambda_{k}}\right) . \\
& +\frac{(\rho / M) \lambda_{1}}{(\rho / M)\left(M-\sum_{j=2}^{L} p_{j}\right) \lambda_{1}-\eta}-\frac{(\rho / M) \lambda_{k}}{(\rho / M) p_{k} \lambda_{k}-\eta}
\end{aligned}
$$

In (15), $\mu^{(n)}$ is the step size at iteration $n$, chosen via a simple inexact line search called a backtracking line search. In the terminology of iterative methods, the convergence of the gradient descent algorithm using the backtracking line search is at least linear [16]. The stopping convergence criteria of an iterative algorithm such as (15) is usually of the form $\|\nabla \psi\| \leq \kappa$, where $\kappa$ is small and positive, as suggested by the suboptimality condition [16]. The stopping criterion is often checked immediately after the descent direction, $-\nabla \psi$, is computed.

The method also requires a suitable starting point $\mathbf{p}^{(0)}$. The objective function in (13) is convex only on the region that the inequality constraints $f_{j}\left(p_{1}, p_{2}, \ldots, p_{L}\right) \leq 0 ; j=1, \ldots, L$ are satisfied. As a result the objective function (14) is not convex over the whole work space $S=\left\{\left(p_{2}, p_{3}, \ldots, p_{L}\right) \in \mathfrak{R}^{L-1} \mid 0 \leq p_{j} \leq M ; p_{1}+\sum_{j=2}^{L} p_{j}=M\right\}$ of the iterative algorithm used here. Since the objective function $\psi\left(p_{2}, p_{3}, \ldots, p_{L}\right)$ may have several local minima, the work space $S$ is divided into $D$ distinct subspaces and the iterative algorithm is run with different starting point candidates chosen from different subspaces. For example, with uniform gridding of the work space and splitting the interval for $p_{j}$ into $q$ equal, distinct segments, we get, at most, $D=q^{L-1}$ subspaces. Then, the converged results are compared to see which one is the global minima. Denoting $\mathbf{p}_{d}^{(c)}$ as the convergent point associated with the starting point chosen from the dth segment, the optimum point allocation is set as

$$
\mathbf{p}^{\mathrm{opt}}=\left[\begin{array}{lll}
p_{1}^{\mathrm{opt}} & p_{2}^{\mathrm{opt}} \cdots & p_{L}^{\mathrm{opt}}
\end{array}\right]^{T}=\left[\begin{array}{ll}
p_{1}^{\mathrm{opt}} & \mathbf{p}_{\mathrm{opt}}^{(c)}
\end{array}\right]^{T}
$$

with

$$
\mathbf{p}_{\text {opt }}^{(c)}=\arg \min _{\substack{(c) \\ d=1, \ldots D}} \psi\left(\mathbf{p}_{d}^{(c)}\right)
$$

In practice, MIMO systems with $N \leq 4$ are of immediate interest, and simulations suggest that for any $N \leq 4, q=N-1$ suffices to avoid local convergence using the above procedure. Finally, we get $C_{\mathbf{H}}=\sum_{i=1}^{L} \log _{2}\left(1+\frac{\rho}{M} p_{i}^{\text {opt }} \lambda_{i}\right)$ and the optimal beamformer $\mathbf{W}^{\text {opt }}$ is obtained from $\mathbf{R}_{\mathrm{ss}}=\mathbf{W} \mathbf{W}^{H} \quad$ and $\mathbf{R}_{\mathrm{ss}}^{\mathrm{opt}}=\mathbf{V} \tilde{\mathbf{R}}_{\mathrm{ss}}^{\mathrm{opt}} \mathbf{V}^{H}$ as $\quad \mathbf{W}^{\mathrm{opt}}=\mathbf{V}\left(\tilde{\mathbf{R}}_{\mathrm{ss}}^{\mathrm{opt}}\right)^{1 / 2}$.

\subsection{Selection from Different Power Allocation Schemes}

In general, the presented optimization in (7) does not maximize the ergodic capacity for all values of system parameters $\rho$, and $\delta_{S N R}$. (this can be seen in the simulations below.) The designer may choose from different power allocation schemes with the metric of ergodic capacity constrained by a maximum allowable SNR loss $\delta_{S N R}$ (in $\mathrm{dB}$ ). The designer picks $\delta_{S N R}$ according to the desired capacity and SER system performances, i.e., capacity and SER are traded off through this parameter. 
The different power allocation schemes for selection are from: the presented optimization; the water-filling schemes applied to different number of eigenchannels, $L \geq 2$; and the dominant eigenmode transmission. The selection procedure is as follows. For each value of $\delta_{S N R}$, the best power allocation scheme, along with the best choice for $L$ that achieves the highest capacity, is selected from the Capacity- $\delta_{S N R}$ plane. This selection guarantees that the average SNR loss on each eigenchannel would be smaller than $\delta_{S N R}$.

\section{SimULATION RESULTS}

To evaluate the performance of the above approach, a $2 \times 2$ and a $3 \times 3$ MIMO system are simulated, but the formulation is applicable to any $N \times M$ MIMO system. In addition, throughout the simulations, the theoretical ergodic capacities of water-filled eigen-MIMO and dominant eigenmode transmission are used as benchmarks.

Figure 2 plots the ergodic capacity (in bits per channel use) versus the SNR loss $\delta_{S N R}(\mathrm{~dB})$ for a $3 \times 3$ MIMO system using the presented optimization applied to different number of eigenchannels, $L \geq 2$, with $\rho=20 \mathrm{~dB}$. Recall that $\delta_{S N R}$ is the maximum allowable SNR loss relative to the average SNR at each receive antenna, imposed on the eigenchannels. It can be verified that for $L \geq 2$, the choice of $\delta_{S N R}$ that can be set within the presented optimization is lower bounded by the one obtained with equal SNRs of all eigenchannels, denoted as $\left.\delta_{S N R}^{\min }\right|_{L}$. The value of $\left.\delta_{S N R}^{\min }\right|_{L}$ depends on various system parameters such as $r=\min (M, N)$ and $L$ (e.g., for a $3 \times 3$ system, $\left.\delta_{S N R}^{\min }\right|_{L=3} \approx+6.08 \mathrm{~dB}$, and $\left.\delta_{S N R}^{\min }\right|_{L=2} \approx-1.8 \mathrm{~dB} \quad$ whereas in a $2 \times 2$ system $\left.\left.\delta_{S N R}^{\min }\right|_{L=2} \approx+3.96 \mathrm{~dB}\right)$.

It is worth noting that in water-filling schemes, a fixed reduction of SNR is imposed on each eigenchannel (this cannot be controlled by the designer) and since the associated SNR loss on the weakest eigenchannel is the largest loss of all the eigenchannels, it can be set as a lower bound for the allowable maximum SNR loss in the water-filling scheme. This sets $\delta_{S N R}(\mathrm{~dB})$ for the water filling case. This is the reason that water-filled capacity curves are the straight lines as seen in Fig. 2. The same situation also holds for the dominant eigenmode transmission

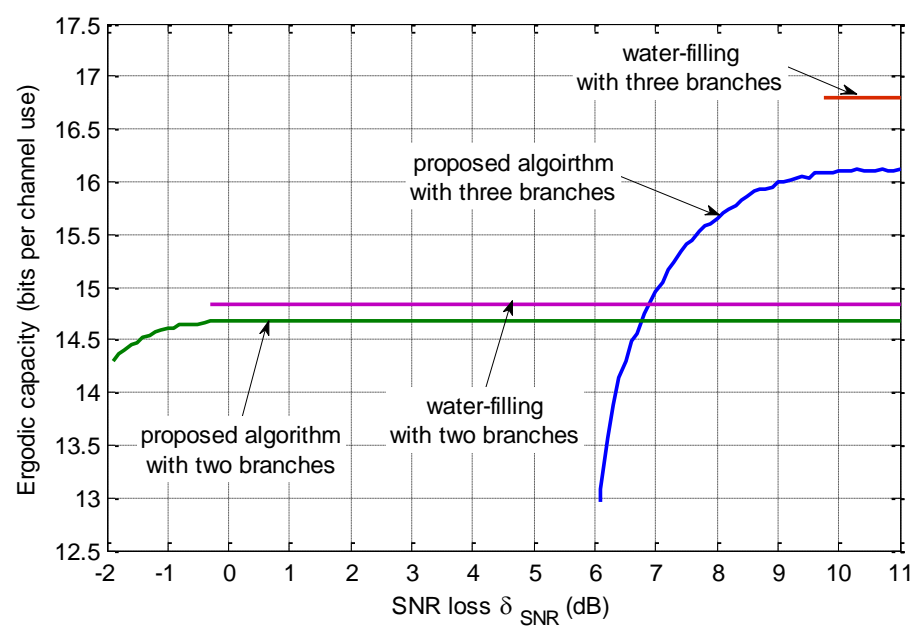

Figure 2. The ergodic capacity versus the SNR loss $\delta_{S N R}(\mathrm{~dB})$ for a $3 \times 3$ MIMO system with $\rho=20 \mathrm{~dB}$ 
(not shown in Fig. 2). The dominant eigenmode transmission has the (fixed) lowest SNR loss of $\delta_{S N R}^{\mathrm{dom}}=-10 \log _{10}\left(\bar{\lambda}_{\max }\right) ; \bar{\lambda}_{\max }=E \lambda_{\max }$, among all schemes.

The capacity results of the presented optimization fall behind the optimal water-filled eigenMIMO capacity for the same $L$. The reason is that, unlike the water-filling scheme that discards the weakest eigenchannel if the power allocated to it is negative, all of the available $L$ eigenchannels are used by the presented optimization all the time. It is seen in Fig. 2 that none of the power allocation schemes maximize the ergodic capacity for all values of $\rho$, and $\delta_{S N R}$. The designer can choose from a number of power allocation schemes (the presented optimization; the water-filling schemes applied to different number of eigenchannels, $L \geq 2$; and the dominant eigenmode transmission) and the metric for selection is the highest ergodic capacity with an allowable maximum SNR loss $\delta_{S N R}$ on eigenchannels. For $\delta_{S N R}^{\mathrm{dom}} \leq \delta_{S N R} \leq\left.\delta_{S N R}^{\min }\right|_{L=2}$, the dominant eigenmode transmission is the only candidate for selection (see Fig. 2).

Figures 3 and 4 illustrate the maximum ergodic capacity achieved via selection, constrained with different choices of $\delta_{S N R}$ for $\rho=0-30 \mathrm{~dB}$ in a $3 \times 3$ and a $2 \times 2$ MIMO systems, respectively. In general, for larger values of $\delta_{S N R}$ (larger loss in SNR is allowed), a system with higher capacity is expected. As a useful feature, the position of the capacity between upper and lower bounds can be controlled by the designer through the choice of $\delta_{S N R}$. Some other interesting results are: making use of more than one eigenchannel, the capacity curves are upper and lower bounded by the optimal water-filled capacity using all eigenchannels $(L=\min (M, N))$ and the one obtained via our approach with $L=2$ and $\left.\delta_{S N R}^{\min }\right|_{L=2}$; in a $2 \times 2$ system, eigenmode transmission is the best for $\delta_{S N R} \leq 6 \mathrm{~dB}$ and values of SNR $\rho<\sim 13 \mathrm{~dB}$; and in a $3 \times 3$ MIMO system, it is possible to outperform the water-filled capacity applied to just the two strongest eigenchannels for any SNR loss $\delta_{S N R} \geq 0 \mathrm{~dB}$.

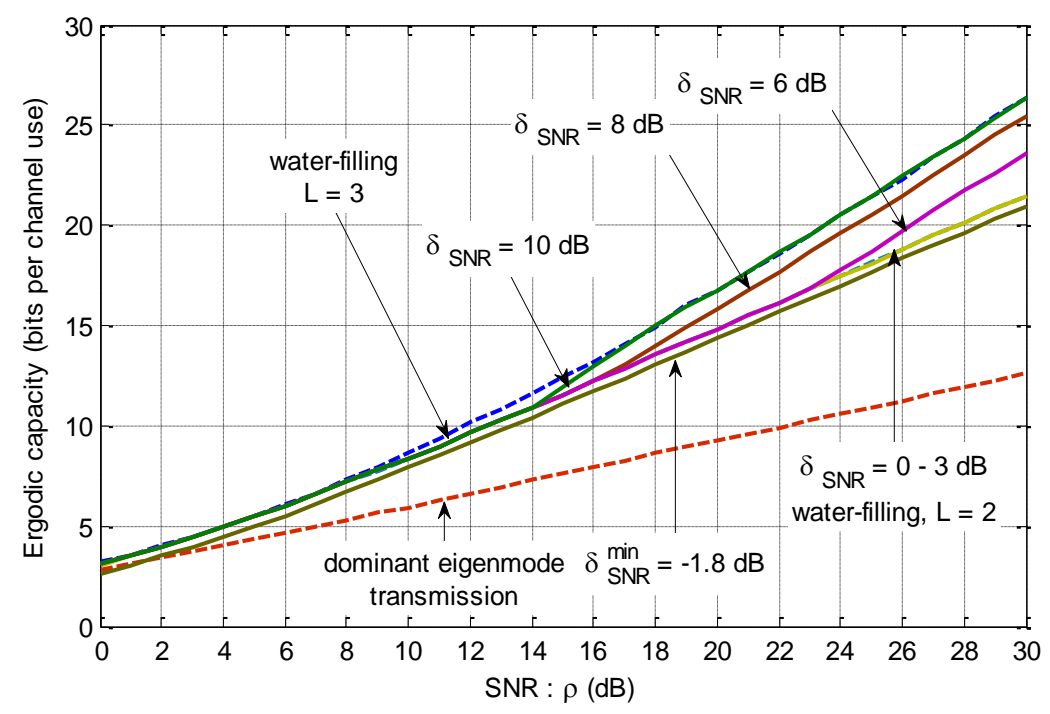

Figure 3 . The maximum ergodic capacity achieved via selection in a $3 \times 3 \mathrm{MIMO}$ system constrained with different choices of $\delta_{S N R}$ 


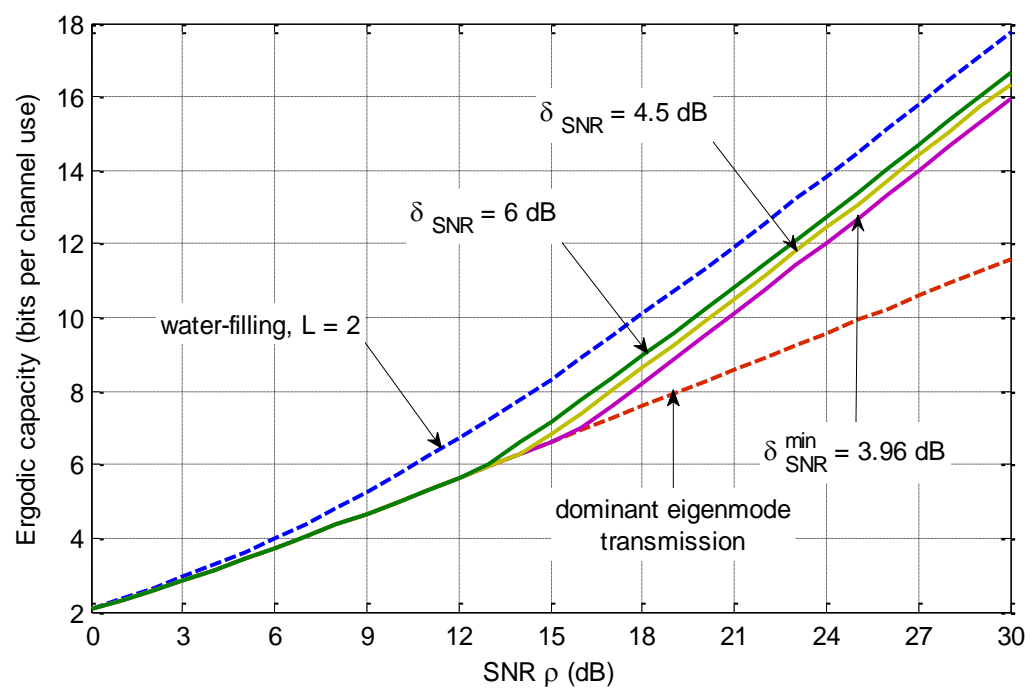

Figure 4 . The maximum ergodic capacity achieved via selection in a $2 \times 2$ MIMO system constrained with different choices of $\delta_{S N R}$

Simulation results are now presented for a $2 \times 2$ MIMO system in which eigenchannel 1 is deployed with independent QAM and eigenchannel 2 is loaded with BPSK. The overall SER performance of the system is a measure for comparison among different power allocation schemes and one definition is

$$
\mathrm{SER} \stackrel{\Delta}{=} \frac{\sum_{i=1}^{r} \# \text { of correctlydetected symbols over eigenchannel } i}{\sum_{i=1}^{r} \# \text { of transmittel symbols over eigenchannel } i}
$$

The above formulation allows the number of eigenchannels in the summations to be smaller than $r$, and in general, the actual number depends on the type of power allocation scheme used. The overall SER results for a $2 \times 2$ system is illustrated in Fig. 5. Also depicted are the SER curves associated with each of the eigenchannels when water-filling is used. These reveal how the overall SER deteriorates with the weakest eigenchannel. In fact, the full eigen-MIMO waterfilling scheme has the worst overall SER performance among other power allocation schemes and the dominant eigenmode transmission has the best. This is expected because of the trade-off between the capacity and SER performances.

Because of the selection aspect of the presented approach, the SER curves are piece-wise discontinuous, and several SER curves for different values of $\delta_{S N R}$ may lie together for some SNR regions. For example, in Fig. 5, the curves tagged with $\delta_{S N R}=6 \mathrm{~dB}, \delta_{S N R}=4.5 \mathrm{~dB}$, and $\delta_{S N R}=3.96 \mathrm{~dB}$ lie on the SER result of dominant eigenmode transmission for low to moderate values of SNR. The promising result is that the overall SER performance is better than that of full eigen-MIMO water-filling. For example, in a $2 \times 2$ system with $\delta_{S N R} \approx 4 \mathrm{~dB}$, the improvement over water-filling is almost $3 \mathrm{~dB}$ in SNR for moderate to high values of SNR ( $\rho \geq \sim 16 \mathrm{~dB}$ ). This improvement is at the expense of a loss of 1.9 bits per channel use in capacity compared to that of water-filling. At low to moderate values of SNR, the advantage is more emphasized - for SER $10^{-3,}$ the equivalent SNR improvement is more than about $15 \mathrm{~dB}$. Here the selection scheme chooses the dominant eigenmode transmission as the best scheme 


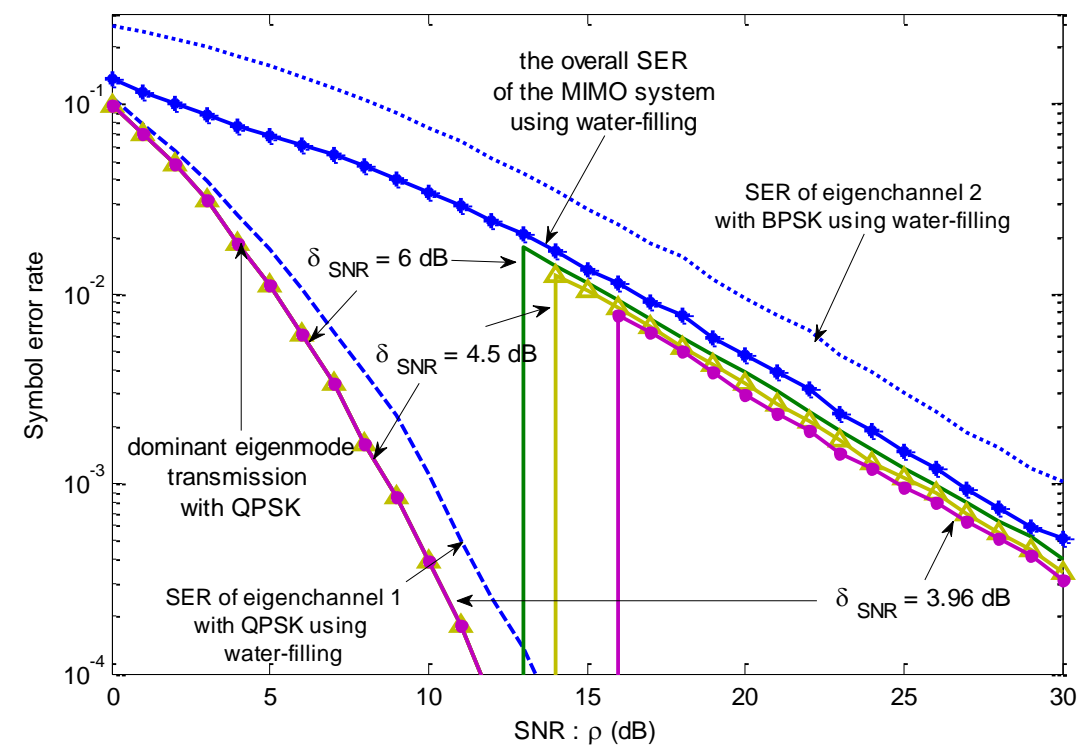

Figure 5. The overall SER results of a $2 \times 2$ system using different power allocation schemes

among others for $\rho<\sim 16 \mathrm{~dB}$ and $\delta_{S N R} \approx 4 \mathrm{~dB}$ and there is only a small loss in capacity (smaller than 1 bit per channel use for $\rho<\sim 10 \mathrm{~dB}$ ) relative to that of water-filling.

\section{SUMMARY AND CONCLUSIONS}

In this paper, the optimal power allocation is formulated for the capacity maximization with the total input power constraint and a maximum allowable SNR loss on eigenchannels, $\delta_{S N R}$. The goal is to find a way to design an eigen-MIMO system that has good reliability, or error performance, and high capacity, simultaneously. Comparison of the ergodic capacities from the presented optimization with that from water-filling with an appropriate number of deployed eigenchannels, and dominant eigenmode transmission, enable selection for optimal capacity for a chosen SNR loss, $\delta_{S N R}$. One feature of the approach is that the SER performance of the system and the position of the capacity between the upper and lower bounds can be controlled by the designer through the choice of $\delta_{S N R}$. Promising results are presented for the improvement of SER performance over than that of full water-filled eigen-MIMO, when using simplified archirectures such as a fixed-set of modulations or fixed common modulation across the eigen-channels. Here, a design can have a controllable and significantly better uncoded error performance than full eigen-MIMO at the expense of a modest loss in capacity. In a practical situation, based on the type of modulation (and any coding) used, the designer may translate the desirable SNR performance to a desired SER performance in a system, and then assign $\delta_{S N R}$ according to the desired capacity and SER system performances.

\section{REFERENCES}

[1] A.Paulraj, R.Nabar and D.Gore, Introduction to space-time wireless communications, Cambridge university press, 2003.

[2] G.Raleigh and J. Cioffi, "Spatio-Temporal Coding for Wireless Communications," IEEE Trans. Commun., Vol 46. No3, March 1998. 
[3] I.E.Telatar, "Capacity of multi-antenna Gaussian channels," European Trans. Telecommun., Vol. 10, No. 6, Nov.-Dec. 1999.

[4] R. Vaughan, and J. Bach Andersen, Channels, Propagation and Antennas for Mobile Communications, The institution of Electrical Engineers, 2003.

[5] J.Yang and S.Roy, "On Joint Transmitter and Receiver Optimization for Multiple-Input-MultipleOutput (MIMO) Transmission Systems." IEEE Trans. Commun., Vol 42. No 12, Dec. 1994.

[6] A.Scaglione,G.B.Giannakis and S.Barbarossa, "Redundant Filter bank Precoders and Equalizers Part I: Unification and Optimal Designs," IEEE Trans. Signal Process., Vol 47, No.7, July 1999.

[7] H.Sampath, P.Stoica, and A.Paulraj, "Generalized linear precoder and decoder design for MIMO channels using the weighted MMSE criterion.” IEEE Trans. Commun., Vol. 49, No. 12, Dec. 2001.

[8] V.Tarokh, N.Seshadri and A.R.Calderbank, "Space-Time Codes for High Data Rate Wireless Communication: Performance Criterion and Code Construction," IEEE Trans. Inform. Theory, Vol 44, No.2, Mar. 1998.

[9] J.Yang and S. Roy, "Joint Transmitter-Receiver Optimization for Multi-Input Multi-Output Systems with Decision Feedback," IEEE Trans. Info. Theory, vol. 40, No. 5, Sep. 1994.

[10] A.Scaglione, S. Barbarossa and G. B. Giannakis, "Filter bank Transceivers Optimizing Information Rate in Block Transmissions over Dispersive Channels," IEEE Trans. Inform. Theory, Vol 45, No.3, Apr. 1999.

[11] B.Mielczarek and W. A. Krzymień, "Comparison of partial CSI encoding methods in multi-user MIMO systems,” J. of Wireless Personal Commun.- Springer, Vol. 52, No. 1, Jan. 2010.

[12] W. Xu,C.Zhao, and Z. Ding, "Optimisation of limited feedback design for heterogeneous users in multi-antenna downlinks,” IET Commun., Vol. 3, No. 11, pp. 1724-1735, Nov. 2009.

[13] T. Yoo and A. Goldsmith, "Capacity and power allocation for fading MIMO channels with channel estimation error," IEEE Trans. Inform. Theory, Vol. 52, No. 5, pp. 2203-2214, May. 2006.

[14] M.Kobayashi,N.Jindal, and G.Caire, "Training and feedback optimization for multiuser MIMO downlink," IEEE Trans. Commun., Vol. 59, No. 8, pp. 2228-2240, Aug. 2011.

[15] A.Svensson, "An introduction to adaptive QAM modulation schemes for known and predicted channels", IEEE Proceedings, Vol. 95, No. 12, Dec. 2007.

[16] S.Boyd, and L.Vandenberghe, Convex Optimization, Cambridge university press, 2004. 